\title{
Recent Advances in Spectroscopy of Hydrogen-Bonded Systems
}

\author{
Marek J. Wójcik, ${ }^{1}$ Paul Blaise, ${ }^{2}$ Joanna Sadlej, ${ }^{3}$ and Henryk Flakus ${ }^{4}$ \\ ${ }^{1}$ Faculty of Chemistry, Jagiellonian University, Ingardena 3, 30-060 Krakow, Poland \\ ${ }^{2}$ Laboratoire "Mathématiques et Physique" (LAMPS), Université de Perpignan, 52 Avenue Paul Alduy, 66860 Perpignan Cedex, France \\ ${ }^{3}$ Faculty of Chemistry, University of Warsaw, Pasteura 1, 02-093 Warsaw, Poland \\ ${ }^{4}$ Institute of Chemistry, University of Silesia, Szkolna 9, 40-006 Katowice, Poland
}

Correspondence should be addressed to Marek J. Wójcik, wojcik@chemia.uj.edu.pl

Received 13 August 2012; Accepted 13 August 2012

Copyright () 2012 Marek J. Wójcik et al. This is an open access article distributed under the Creative Commons Attribution License, which permits unrestricted use, distribution, and reproduction in any medium, provided the original work is properly cited.

Hydrogen bond plays a crucial role in many areas of physics, chemistry, and biology. This issue compiles five exciting papers presenting the state of the art in spectroscopic and computational aspects of hydrogen-bonded systems in three original research articles and two reviews.

These papers will stimulate the continuing efforts to understand molecular interactions in hydrogen-bonded complexes, liquids and solids, the development of experimental and theoretical strategies to treat hydrogen-bonded systems, and the new evaluation of outcomes.

The papers describe new methods for characterization of spectra, advances in molecular description of molecular interactions in hydrogen-bonded systems, new insights into molecular models, and current concepts in the treatment of small and large hydrogen-bonded systems. The topics include recent developments in experimental and theoretical studies of hydrogen-bonded systems, advances in theoretical models of hydrogen bonds, spectroscopy of hydrogen bonds in excited electronic states, proton transfer in hydrogenbonded complexes, photophysics of hydrogen-bonded systems, polymorphism, and dynamical aspects of hydrogen bonding.

In the paper by A. Gorski et al., the authors discuss polymorphism, hydrogen bond properties, and vibrational structure of $1 H$-pyrrolo[3,2- $h$ ] quinoline dimers performing analysis of IR and Raman spectra, combined with DFT calculations. Theoretical investigation of the cooperativity in the $\mathrm{CH}_{3} \mathrm{CHO} \cdot 2 \mathrm{H}_{2} \mathrm{O}, \mathrm{CH}_{2} \mathrm{FCHO} \cdot 2 \mathrm{H}_{2} \mathrm{O}$, and $\mathrm{CH}_{3} \mathrm{CFO} \cdot 2 \mathrm{H}_{2} \mathrm{O}$ systems is presented in the paper by A. K. Chandra and T. Zeegers-Huyskens. Temperature and H/D isotopic effects in the IR spectra of the hydrogen bond in solid-state 2-furanacetic acid and 2-furanacrylic acid are discussed in the paper by H. Flakus and A. Jarczyk.

L. Sobczyk et al. review proton transfer equilibria and critical behavior of $\mathrm{H}$-bonding. They analyze the hydrogen bond properties of the acid-base systems depending on the ability of the proton transfer in the formulation of the Brönsted approach. M. J. Wójcik et al. present theoretical studies of dynamic interactions in excited states of hydrogenbonded systems. They present model for vibrational interactions in the hydrogen-bonded dimer of benzoic acid in electronically excited state which is used for theoretical simulation of the $\mathrm{O}-\mathrm{H}$ stretching IR absorption bands and calculate tunneling splittings for vibrationally excited states in electronically excited tropolone.

By compiling these papers, we hope to enrich our readers and researchers with respect to the modern problems of spectroscopy of hydrogen-bonded systems.

Marek J. Wójcik Paul Blaise Joanna Sadlej Henryk Flakus 

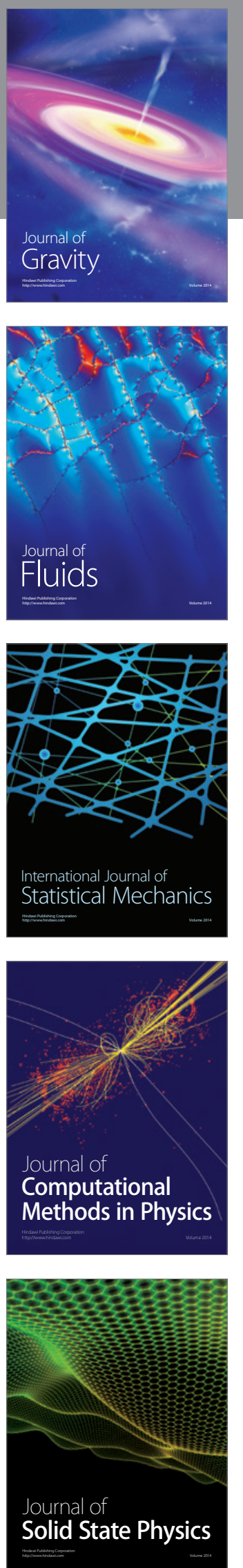

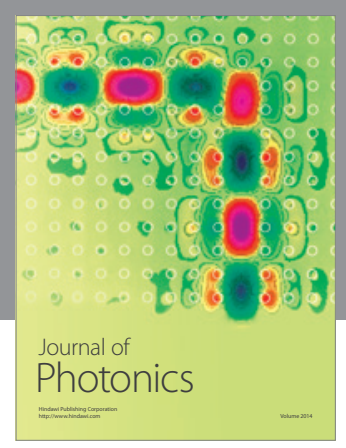

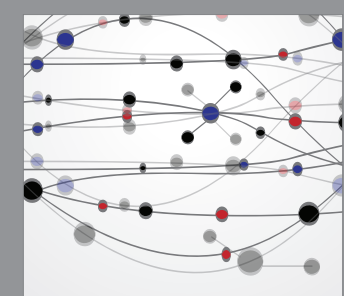

The Scientific World Journal
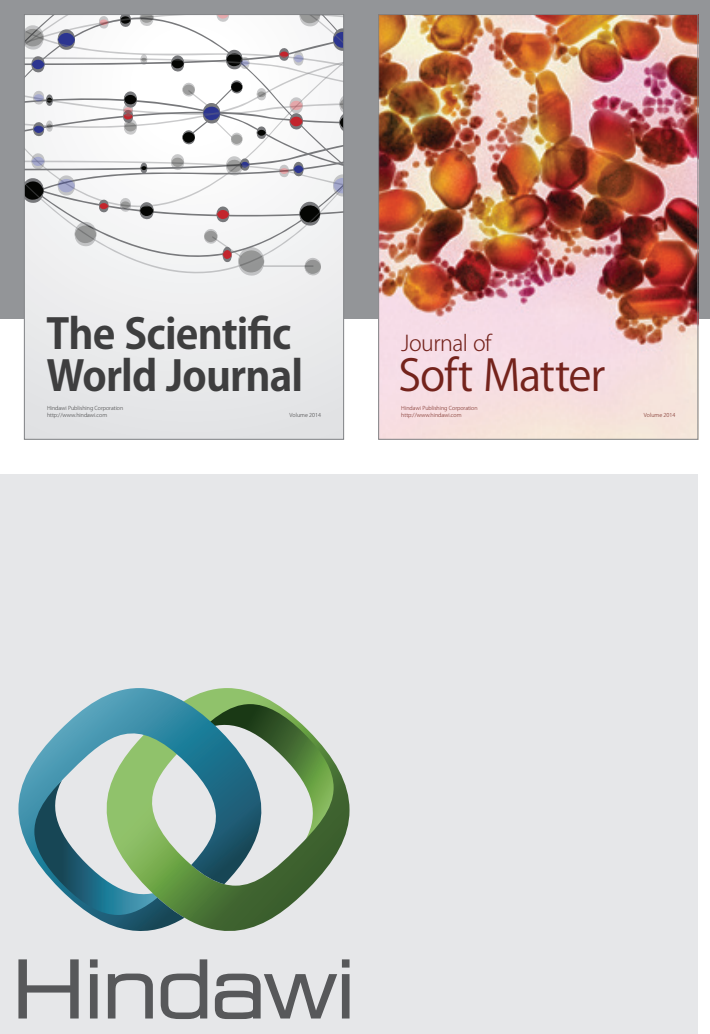

Submit your manuscripts at

http://www.hindawi.com
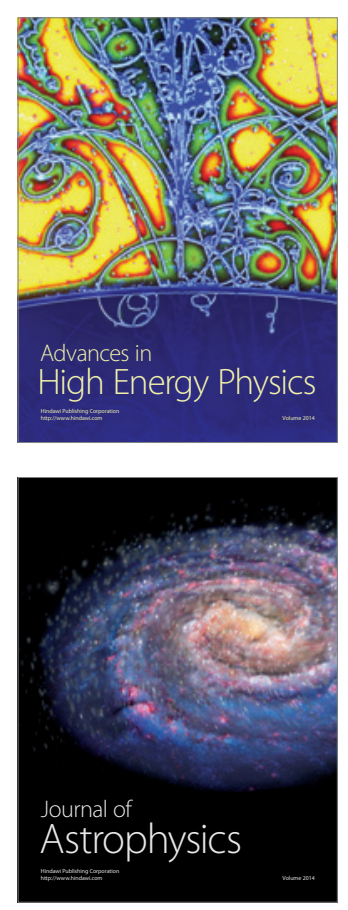
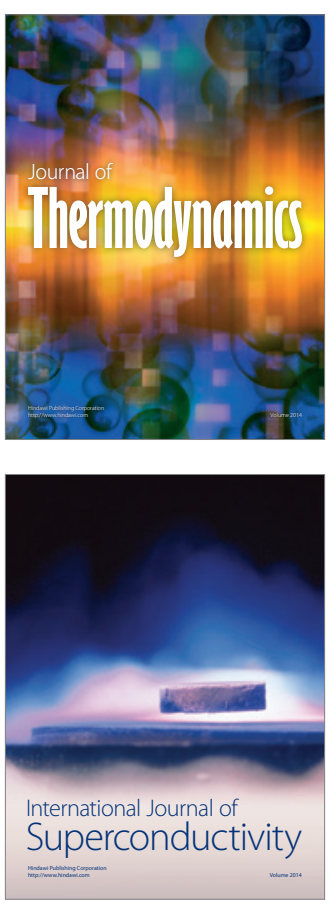
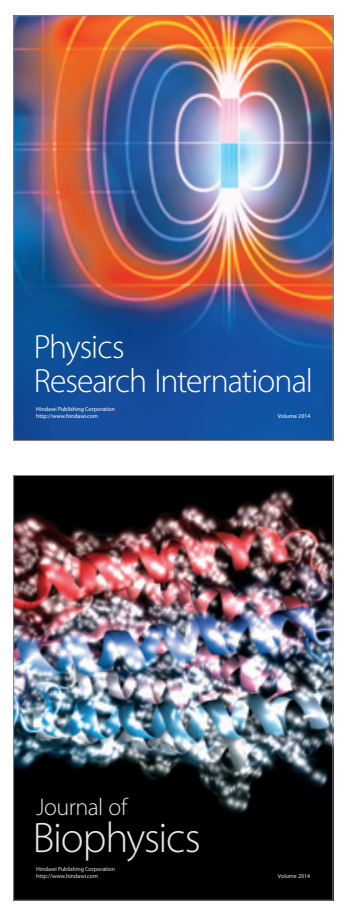
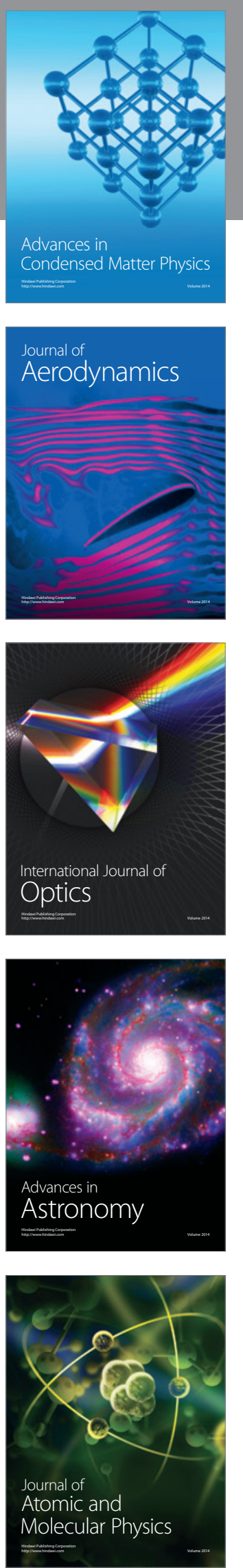